\title{
Experimental study on evolution law for particle breakage during coal and gas outburst
}

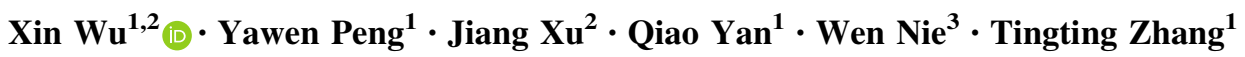

Received: 17 July 2019/Revised: 4 September 2019/Accepted: 8 November 2019/Published online: 26 November 2019

(C) The Author(s) 2019

\begin{abstract}
Coal and gas outburst is a dynamic phenomenon in underground mining engineering that is often accompanied by the throwing and breakage of large amounts of coal. To study the crushing effect and its evolution during outbursts, coal samples with different initial particle sizes were evaluated using a coal and gas outburst testing device. Three basic particle sizes, 5-10 mesh, 10-40 mesh, and 40-80 mesh, as well as some mixed particle size coal samples were used in tests. The coal particles were pre-compacted at a pressure of $4 \mathrm{MPa}$ before the tests. The vertical ground stress (4 MPa) and the horizontal ground stress $(2.4 \mathrm{MPa})$ were initially simulated by the hydraulic system and maintained throughout. During the tests, the samples were first placed in a vacuum for $3 \mathrm{~h}$, and the coal was filled with gas $\left(\mathrm{CH}_{4}\right)$ for an adsorption time of approximately $5 \mathrm{~h}$. Finally, the gas valve was shut off and the coal and gas outburst was induced by quickly opening the outburst hole. The coal particles that were thrown out by the outburst test device were collected and screened based on the particle size. The results show the following. (1) Smaller particle sizes have a worse crushing effect than larger sizes. Furthermore, the well-graded coal particles are weakly broken during the outburst process. (2) As the number of repeated tests increases, the relative breakage index grows; however, the increment of growth decreases after each test, showing that further fragmentation becomes increasingly difficult.
\end{abstract}

Keywords Coal and gas outburst $\cdot$ Particle size $\cdot$ Breakage $\cdot$ Gradation $\cdot$ Adsorption

\section{Introduction}

Coal and gas outburst is a type of dynamic phenomenon in underground mining processes. Over a relatively short time (a few seconds to a few minutes), coal (or rock) and gas (or $\mathrm{CO}_{2}$ ) is ejected at high speeds, which always leads to casualties and/or economic losses (Zhang 2008; Hu et al.

Xin Wu

xinwu@sicnu.edu.cn

1 College of Engineering, Sichuan Normal University, Chengdu 610101, China

2 College of Resources and Environmental Science, Chongqing University, Chongqing 400030, China

3 Quanzhou Institute of Equipment Manufacturing, Haixi Institutes, Chinese Academy of Sciences, Quanzhou 362000, China
2007). Considering the complexity of this mechanism and the diversity of outburst phenomena, a variety of coal and gas outburst mechanism hypotheses have been proposed. The most representative hypothesis is the ground stress hypothesis, which emphasizes that the energy of the coal and gas outburst comes from the elastic deformation energy that accumulates in the rock around the coal seam. The gas effect hypothesis emphasizes that gas is the main source of the outburst energy. The chemical nature hypothesis considers that the outburst is caused by high pressures and that heat has formed from the chemical reactions. The comprehensive hypothesis is considered to be the result of the combined effects of the ground stress, gas, and physical and mechanical properties of the coal. The most representative of the comprehensive hypotheses is the inhomogeneous ground stress hypothesis proposed by Pavlov of the former Soviet Union Marcoyev Institute 
and the energy hypothesis proposed by B.B. Hodort (Zhou and Lin 1999). The latter believes that coal and gas outbursts are caused by the deformation potential of the coal and gas inner energy. Furthermore, when the stress state of the coal seam changes suddenly, the released potential energy causes the coal body to be damaged with an associated gas desorption, called the energy balance hypothesis of gas-bearing coal seams. In recent decades, physical simulation experiments and theoretical numerical analyses have been widely applied to research on coal and gas outbursts (Yang et al. 2018; Gupta et al. 2017).

Based on the conservation of energy, a large amount of elastic energy for the coal rock mass and gas internal energy is released during the outburst process. This energy is converted into crushing and transport work of the coal, as well as energy in the form of friction heat, vibration, etc. (Xie et al. 2010; Wei et al. 2010). Due to the complexity of experimental studies on the crushing and transport work of coal and gas outbursts, several studies have focused primarily on theoretical models (Zhao et al. 2010; Hu et al. 2008; Jiang and Yu 1996). For example, elastic mechanics has been used to analyze the initiation, preparation, and development of coal and gas outbursts, the potential of coal seams, the kinetic energy of the surrounding rock, and the internal energy of the gas.

After the actual coal and gas outburst, coal particles generally have a high degree of fragmentation and a wide size distribution. Research on coal particle size degradation and the generation of fine particles has been reported in the literature. Under the same energy conditions, the coal lithotype (Shi et al. 2018), particle sphericity (Zhou et al. 2017), initial particle size (Oberholzer and van der Walt 2009), and proportion of fine particles (Xie et al. 2017) all affect the breakage rate of coal particles. That is, the amount of energy required for breakage is predominantly dependent on the size, shape, and breakage properties of the specimen as well as the input energy (Sahoo 2005, 2006; Sahoo and Roach 2005).

The size degradation depends on the cumulative breakage energy imparted to the coal and its inherent strength. While the bulk material strength of coal is dependent on its rank and composition, the relative strengths of the individual coal particles are also dependent on their size (Esterle et al. 2002). For all materials, the energy required for size reduction is proportional to the newly created surface area; hence, more energy is required for a smaller parent to achieve the equivalent breakage as a larger parent. It is well known that the crack density of larger particles is much greater than that for smaller particles (Shi 2016; Krajcinovic and Mastilovic 1995; Tavares and King 1998). Therefore, larger particles tend to be weaker and easier to break.
The above research focused primarily on the mechanical crushing of coal and rock. However, research on coal particle crushing in coal and gas outburst processes is lacking. To understand the mechanism of coal and gas outbursts as well as having a verification and supplement to theoretical models, a large-scale test device for coal and gas outburst simulations is used to test the crushing and transport work of coal samples (Xu et al. 2008). In this paper, the fracture effect and variation law of outbursts with different initial particle sizes and the same test samples under multiple outburst tests are studied.

\section{Energy conservation during coal and gas outburst}

If coal and gas outbursts are treated as mechanical processes, coal must be subjected to kinetic forces; however, the coal body also generates a resistance. An outburst occurs when the resistance is less than kinetic force. If only the relationship between different forces is considered, it is difficult to obtain a systematic and comprehensive understanding of the various factors that constitute the mechanical system as it is a complex and variable system. The effects of various forces, which help predict coal and gas outbursts, can be calculated quantitatively through tests when the actions of the outburst force and energy are considered.

\subsection{Energy relationship}

The energy sources of the outburst process are from the ground stress elastic potential and the gas expansion. The coal and rock mass are the receptors of the dynamics and the main body of resistance during the entire process. Considering the conservation of energy, the resistance of the coal body is equal to the work done by the external force to destroy it (crushing work). The energy consumed when a coal body is thrown out is equal to the transport work done by the external force during the outburst process. Therefore, in a mechanical system that consists of coal and gas, there are four energy relationships that interact and transform with each other. When the gas and ground stress potential is greater than the crushing and transport work of the coal body, an outburst can occur. That is, the condition for a coal and gas outburst can be approximated as:

$W_{1}+W_{2}>W_{A}+W_{y}$

where $W_{1}$ is the gas expansion energy, $W_{2}$ is the elastic potential of the coal body, $W_{A}$ is the crushing work, and $W_{y}$ is the transport work. 
The situ tests show that the elastic potential in the coal body is generally smaller than the expansion energy of the coal seam gas (Wei et al. 2010). This is because the magnitude of the energy is simultaneously affected by the stress and strain. Although the gas pressure of the coal seam is smaller than the ground stress, it has a larger expansion. In the end, the strain of the gas internal energy is larger than the ground stress potential so the gas expansion can release more energy than the ground stress. Zheng confirmed these conclusions from the underlying physical mechanism, and believed that the gas internal energy is the main provider of outburst energy. In addition, the gas potential in the outburst coal seam is 1-3 orders of magnitude larger than the elastic potential of the coal body (Zheng 2004).

\subsection{Energy sources}

\section{(1) Gas expansion energy}

A variety of energy balances are obtained from detailed studies on gas expansion (Zhao et al. 2010; Hu et al. 2008; Jiang and Yu 1996). As the outburst process occurs rapidly, there is no time for heat exchange for the coal-gas system with the outside environment; therefore, the outburst process is simplified as an adiabatic process. The expansion work of the gas in adiabatic expansion processes is readily obtained from the equation of state and the process equation of gas (Zhao et al. 2010):

$W_{1}=\int d W=\frac{1000 p_{1} V}{n-1}\left[\left(\frac{p_{2}}{p_{1}}\right)^{\frac{n-1}{n}}-1\right]$

where $W_{1}$ is the work done by gas expansion energy in $\mathrm{kJ} / \mathrm{t}$, $P_{1}$ and $P_{2}$ are the environmental pressure and outburst coal seam gas pressure in MPa, respectively, $n$ is the adiabatic index of gas taken as 1.31 , and $V$ is the gas volume participate in the outburst in $\mathrm{m}^{3}$.

(2) Coal body elastic potential

When the stress state of the coal seam near the working face changes abruptly, the potential of the coal body may be suddenly released. The amount of potential released depends on the stress state before and after the outburst of the working face. In fact, the coal body is affected by mining, so the stress state constantly changes. The breaking and throwing of the coal body experiences two processes: the original stress state to the stress concentration state and the stress balance to the limit equilibrium state. A portion of the potential energy is transformed into coal crushing work. When the coal body is subjected to hydrostatic pressures, the potential of the coal body is (Wen 2003):

$W_{2}=10^{3}(1-2 \mu) \sigma_{0}^{2} /(2 E \gamma)$ where $\mu$ is Poisson's ratio of the coal body, $\sigma_{0}$ is the original stress of coal in $\mathrm{MPa}, E$ is the elastic modulus of the coal body in MPa, and $\gamma$ is the coal body density.

\subsection{Energy release}

\section{(1) Crushing work}

An essential feature of coal and gas outburst is the coal that is thrown out becomes highly fragmented with a significant amount of coal powder. A large portion of the energy is consumed in the broken coal. The study of the relationship between the fracture size and fracture work for the rock includes the new surface theory, similar theory, and crack theory (Guo et al. 2000).

The new surface theory assumes that the only difference before and after an object broken is that new surface area is added, and the energy required to produce the new surface is directly proportional to the crushing work. Geometrically-similar solid particle volumes are proportional to the cube of its linear dimension, whereas the area is proportional to its square. Therefore, the surface area per unit volume is inversely proportional to its geometric size. The results of microscopic studies show that coal particle outbursts are primarily spherical particles. The surface area of spherical particles per unit volume is

$S=4 \pi\left(\frac{d}{2}\right)^{2} / \frac{4}{3} \pi\left(\frac{d}{2}\right)^{3}=6 \frac{1}{d}=K \frac{1}{d}$

where $d$ is the geometrically-similar solid particle diameter and $K$ is a constant that depends on the particle shape.

Based to the new surface theory, the work consumed during coal rock crushing is proportional to the new surface added after crushing. Therefore, the work required to crush a unit volume of coal rock is

$W_{A}=K_{R} \cdot(1 / d-1 / D)$

where $K_{R}$ is a constant that depends on the nature of the rock sample and its breaking mechanism, $D$ is the original size of the rock sample, and $d$ is the size after the rock sample is broken.

Similar theory considers that when a rock sample reaches a critical value under an external load, the crushing work to break a piece into a smaller piece is constant. Thus, the crushing work is considered to be:

$W_{A}=K_{k} \cdot[\lg (1 / d)-\lg (1 / D)]$

where $K_{k}$ is a constant that depends on the nature of the rock sample and its breaking mechanism.

Crack theory considers various cracks that are hidden in the rock, indicating the rock crushing work should be: 
$W_{A}=K_{B} \cdot(1 / \sqrt{d}-1 / \sqrt{D})$

where $K_{B}$ is a constant that depends on the nature of the rock sample and its breaking mechanism.

\section{(2) Transport work}

The transport work refers to the kinetic energy of the broken coal when it is thrown out during an outburst. This work is related to the mass of the outburst coal and its initial velocity as:

$W_{v}=\frac{1}{2} m v_{0}^{2}$

where $m$ is the mass of the coal and $v_{0}$ is its initial velocity.

Since the initial velocity of outburst coal is difficult to measure, the above formula cannot be used to directly calculate the transport work of broken coaly. Thus, some scholars (Wen 2003) considered that the work of the broken coal under ideal conditions is:

$d W_{v}=x g\left(f_{1} \cos \alpha \pm \sin \alpha\right) d m$

where $x$ is the moving distance, $f_{1}$ is the drag coefficient, $\alpha$ is the roadway inclination, and $m$ is mass of coal that is thrown out.

\section{Test materials and methods}

\subsection{Test materials}

Ordinary bituminous coal was used as the test material for the experiments. The coal sample was taken from the No. 8 coal seam of the Datong Mine of Chongqing Energy Co., Ltd., which is located at the junction of the Yungui Plateau and Sichuan Basin. The well field structure is an anticline with a gentle dip angle. The No. 8 is the primary coal seam has an average thickness of $2.75 \mathrm{~m}$. The lithology of the roof is mudstone, siltstone, and sandy mudstone, there is a large pressure on the roof of the working face, the coal seam is loose and broken, and this mine experiences significant coal and gas outbursts.

Industrial analyses were performed to better understand the physical and mechanical properties of the coal samples. The results are shown in Table 1.

\subsection{Test device and methods}

The test coal sample was the same as the industrial analysis coal sample that was collected from the No. 8 coal seam of the Datong Mine. To study the effects of the particle size on the crushing and transportation work during coal and gas outburst processes, the original coal sample was mechanically crushed, and coal particles of different sizes
Table 1 Industrial analyses of the coal sample

\begin{tabular}{lllllll}
\hline Parameter & $M_{\mathrm{ad}}$ & $A_{\mathrm{d}}$ & $V_{\mathrm{daf}}$ & $\mathrm{TRD}$ & $\mathrm{ARD}$ & $\mathrm{F}$ \\
\hline Values & $1.00 \%$ & $12.43 \%$ & $12.43 \%$ & 1.49 & 1.35 & $9.40 \%$ \\
\hline
\end{tabular}

Table 2 Test plan and number

\begin{tabular}{lllllll}
\hline Coal particle size & I-1 & I-2 & I-3 & II-1 & II-2 & II-3 \\
\hline 5-10 mesh & 1 & 0 & 0 & 0 & $1 / 3$ & \\
$10-40$ mesh & 0 & 1 & 0 & $1 / 2$ & $1 / 3$ & None $^{a}$ \\
$40-80$ mesh & 0 & 0 & 1 & $1 / 2$ & $1 / 3$ & \\
\hline
\end{tabular}

${ }^{\mathrm{a} C o a l}$ sample is directly from the mechanical crushing of larger pieces

were sieved. The test plan and the number of different experimental groups are shown in Table 2.

Laboratory simulations are among the most effective methods to study coal and gas outbursts. The Institute of Mechanics of the Chinese Academy of Sciences, China University of Mining and Technology and Henan Polytechnic University have conducted research in this field and developed corresponding testing devices. In this paper, the coal and gas outburst test device developed by Chongqing University (Xu et al. 2008; Nie et al. 2016) was used to conduct the coal and gas outburst testing ( $\mathrm{Wu}$ 2010). The device is composed of an outburst assembly, fast-releasing component, load-bearing frame, electric servo-controlled loading system, reversal unit, main frame bracket, and accessories. The test equipment and principle of operation are shown in Fig. 1.

The main testing steps are as follows:

(1) A sealable metal box is used as the carrier, the coal particles are layered and compacted at a pressure of $4 \mathrm{MPa}$, as shown as Fig. 1b, and approximately $100 \mathrm{~kg}$ of coal particles is required for each test. In addition, temperature and pressure sensors are required during testing, while the enclosure is sealed, as shown in Fig. 1c.

(2) The test box is lifted to the coal and gas outburst test device and affixed to it. At the start of test, the vertical ground stress $(4 \mathrm{MPa})$ and the horizontal ground stress (2.4 MPa) are simulated using the hydraulic system, as shown in Fig. 1d.

(3) The coal sample in the sealed box is evacuated to a predetermined negative pressure before adding gas $\left(\mathrm{CH}_{4}\right)$ to saturate the coal body under a $1 \mathrm{MPa}$ gas pressure. The adsorption process is performed for not less than $5 \mathrm{~h}$, while the temperature, pressure and 

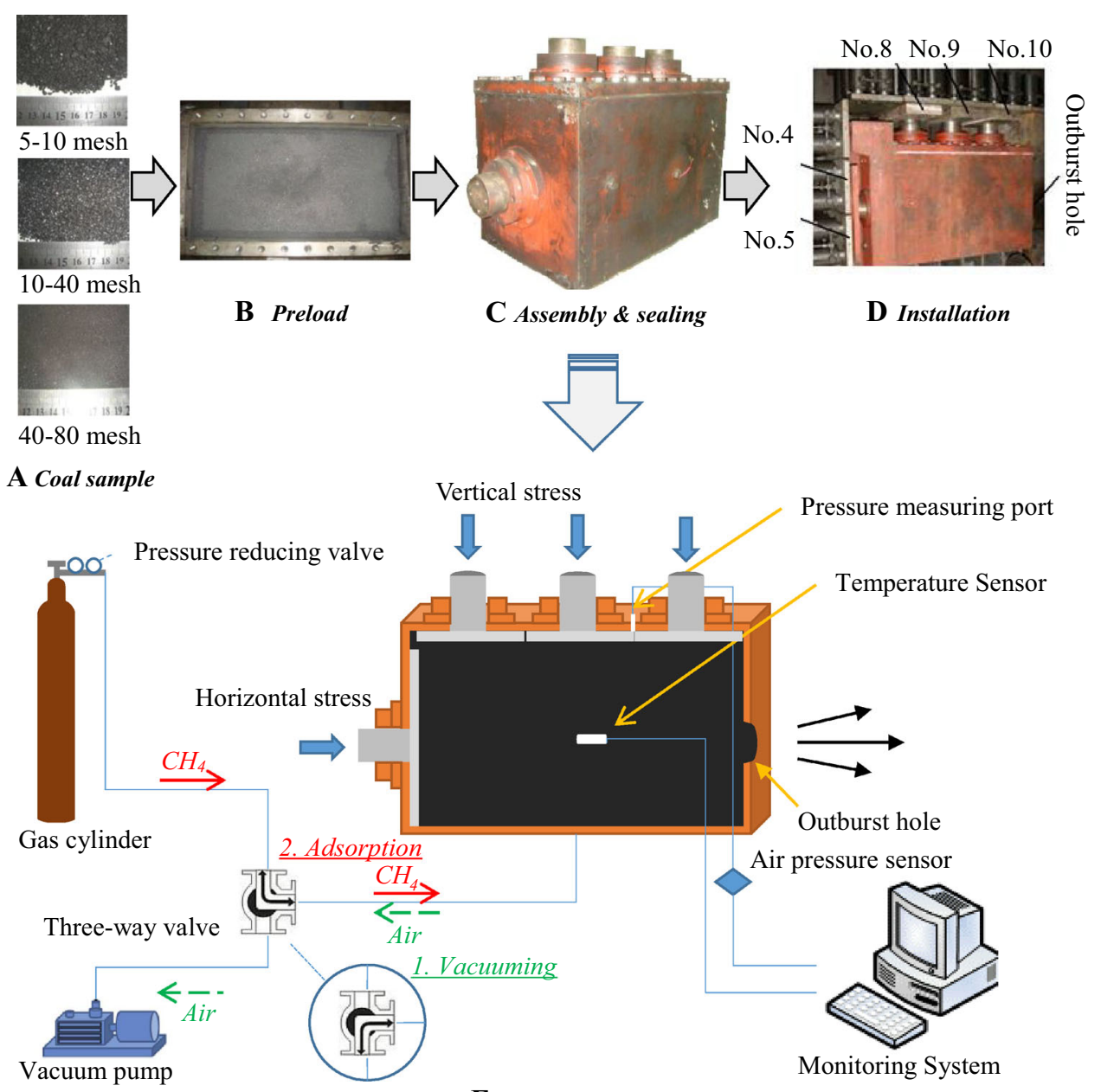

E Test device schematic

Fig. 1 Coal and gas outburst simulation test device

other signals from the process are recorded, as shown in Fig. 1e.

(4) The adsorption is completed and the simulated outburst process is begun. The gas valve is turned off and the coal and gas outburst is induced by quickly opening the outburst hole. Then, the coal and gas are sprayed at a high speed.

(5) The test is stopped. After the gas volume concentration at the site is less than $1 \%$, the coal is collected and sieved, while the shape and volume of the outburst cavity are measured.

After the above experiments, the basic laws for changes in the pressure and temperature are obtained. The results show that the coal body temperature is closely related to the gas adsorption and desorption processes, and that coal particles with smaller sizes have faster gas adsorption and desorption rates. The test process can be roughly divided into three stages:
(1) The vacuuming stage which lasts for approximately 2 to $3 \mathrm{~h}$. At this stage, the temperature of the coal body slowly decreases, where the total decrease is about $0.45-0.6{ }^{\circ} \mathrm{C}$. Furthermore, the temperature drop of the coal samples with smaller particle sizes, such as Test I-3, is faster and to a greater extent than for larger particles.

(2) The gas adsorption stage After the entering gas has a pressure of $1 \mathrm{MPa}$, the temperature in the tank rises sharply during the first $100 \mathrm{~s}$, which can reach $1-3{ }^{\circ} \mathrm{C}$. Then, the temperature increase rate of the coal body gradually slows to its equilibrium state. In addition, the temperature rise for the coal samples with smaller particle sizes, such as Test I-3, is faster and to a greater extent than for larger particles.

(3) The outburst stage The outburst hole is opened quickly. When coal and gas outburst occurs, the gas pressure drops rapidly, and the temperature of the coal body decreases by $0.2-0.5^{\circ} \mathrm{C}$ instantly. Then, the temperature drop slows and eventually balances out. 


\section{Results and analysis}

\subsection{Overview of results}

For Test I-2 (10-40 mesh), the mass of the coal thrown out from the metal box is $16.83 \mathrm{~kg}$, while the initial mass is $86.65 \mathrm{~kg}$. The relative outburst strength, which is the ratio of the outburst coal mass to the total coal mass, is $19.43 \%$. Relying on the three different particle size tests, it was found that the mass of the outburst coal for Test I-1 (5-10 mesh) is $5.78 \mathrm{~kg}$, and the relative outburst strength is $6.47 \%$, while Test I-3 has $20.24 \mathrm{~kg}$ of outburst coal and a relative outburst strength of $23.25 \%$. When the coal and gas outbursts occur, the energy released from the small particle coal samples is more intense, which is consistent with the adsorption of more gas and the accumulation of more gas energy in the small particle coal samples. The tests for mixed coal particles sizes, such as Tests II-1, II-2 and II-3, are more complicated, but follow this same basic rule.

According to the measurements of the coal thrown out by the test device, the particle size of the coal after the outburst is reduced to different extents, which reflects the crushing work of the process. In addition, the coal is thrown to different distances, which reflects the variability in the transport work. To analyze the above vital parameters in detail, the laboratory area is divided into regions, and the particle size variation and distribution laws for the coal are analyzed by region, as shown in Fig. 2.

\subsection{Different initial particle size test}

The gradation curve of each area before and after the outburst and the mass of coal in each area are shown in Fig. 3. The results show that there is an apparent regional distribution for the coal particles thrown out during the outburst process. The crushing work of the coal particles in various regions is different, and the crushing and transport work is strongly influenced by the particle size of the initial coal sample.

The distribution of coal samples with smaller particle sizes is further from the outburst hole, which indicates a greater release of the transport work. Test I-1 samples (510 mesh) are concentrated primarily in the No. 1 area, which is close to the outburst hole. In comparison, Test I-3 samples (40-80 mesh, fine particles) are located primarily at the No. 3 area, which is farther from the outburst hole. The results of Test I-2 are somewhere in between. This phenomenon can be explained by the energy formula of Eq. (1). For coal samples with smaller particle sizes, more gas can be absorbed, which accumulates more gas internal energy $W_{1}$ over the same limited time, which leads to a

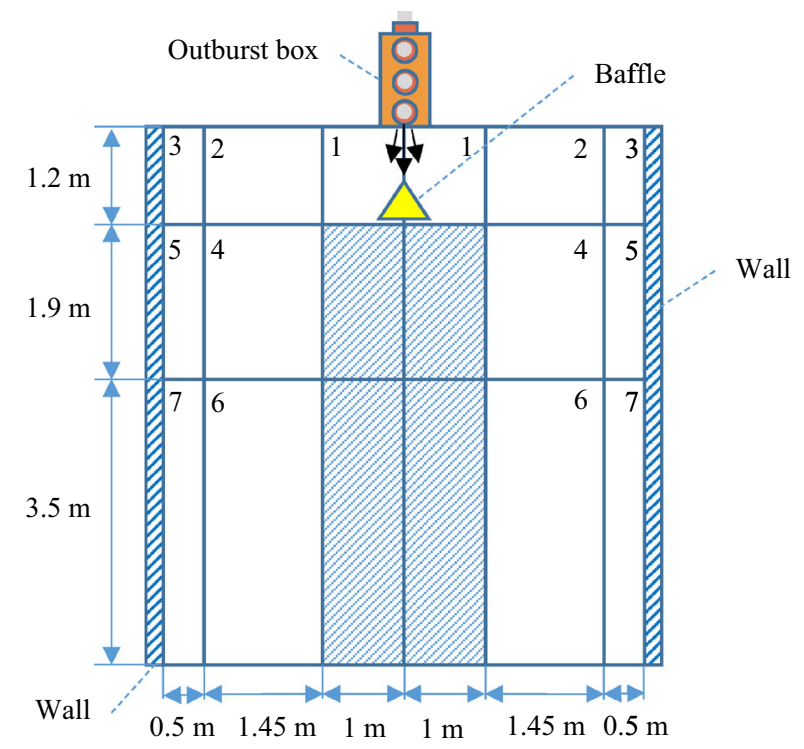

Fig. 2 Regions where coal is collected and analyzed (top view)

greater release of crushing and transport work. As a result, the coal particles are sprayed to greater positions.

A suitable fracture index is introduced to compare the crushing work of coal particles of different sizes. The relative breakage index, which is the ratio of the amount of crushing to the potential, was defined by Hardin (1985) as an indicator and is used here, as shown in Fig. 4.

$B_{r}=\frac{B_{t}}{B_{p}}$

where $B_{r}$ is the relative breakage and is between 0 and $1, B_{t}$ is the area enclosed by three points A, C, D (yellow part in Fig. 4) and represents the amount of crushing, and $B_{p}$ is the area enclosed by three points $\mathrm{A}, \mathrm{B}, \mathrm{D}$ (diagonal part in Fig. 4) and represents the breakage potential. Therefore, a smaller $B_{r}$ leads to a worse crushing effect.

The relative breakage $B_{r}$ of particles with different initial particle sizes was analyzed, as shown in Fig. 5. For the three basic particle size tests, Test I-1, Test I-2, and Test I3 , smaller particle sizes had less crushing work than larger particles. In addition, the gradation curves for the mixed particle sizes (Test II-1, Test II-2, and Test II-3) show that coal with a good grading has a worse crushing effect. Specifically, the $B_{r}$ of Test II-3 is the smallest of the three tests.

\subsection{Cumulative crushing test}

The influence of multiple tests on the crushing effect of coal and gas outbursts is analyzed with a focus on Test II-3. The laboratory area is divided into five rectangular regions (Areas A to E) that are aligned linearly. The coal sample for this test is directly from the mechanical crushing of 


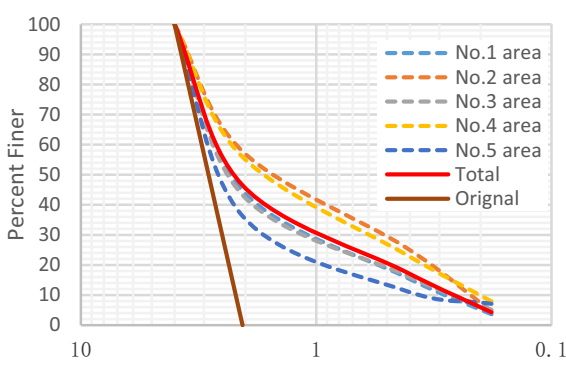

Particle diameter ( $\mathrm{mm}$ ) A Test I-1 gradation curve

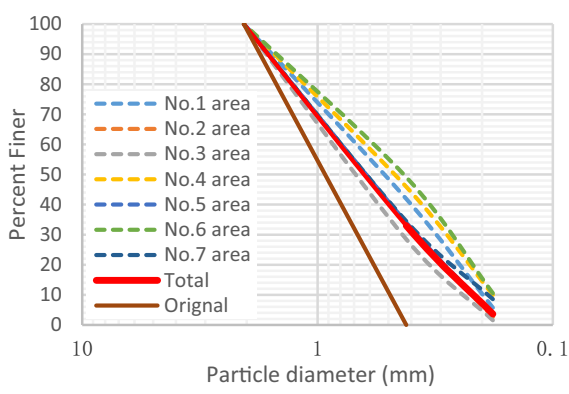

C Test I-2 gradation curve
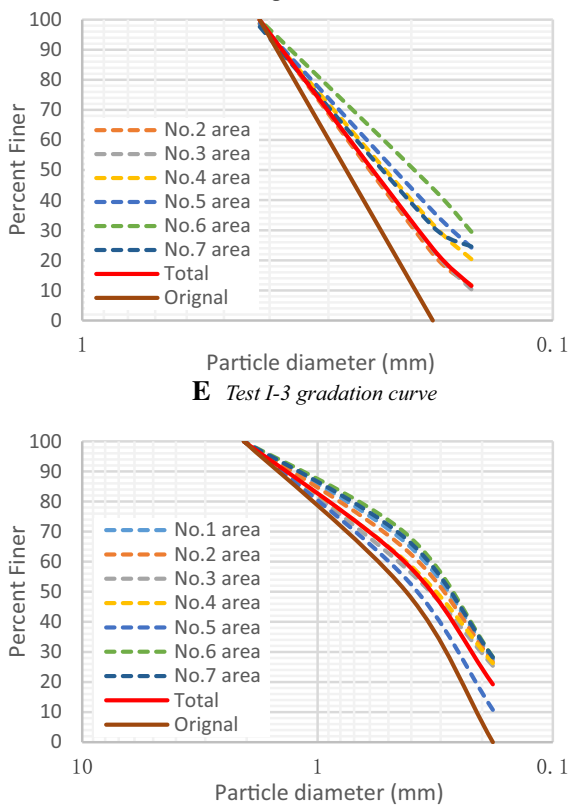

G Test II-1 gradation curve

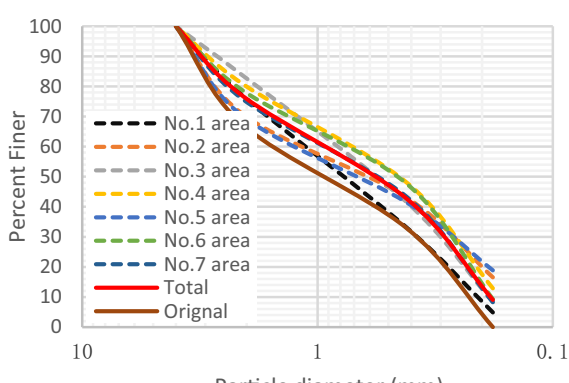

Particle diameter $(\mathrm{mm})$
I Test II-2 gradation curve

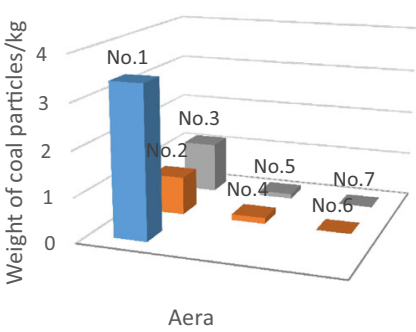

B Test I-1 Coal particle distribution

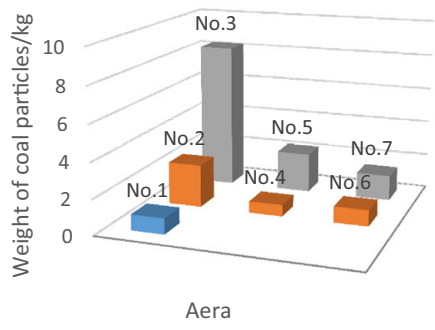

D Test I-2 Coal particle distribution

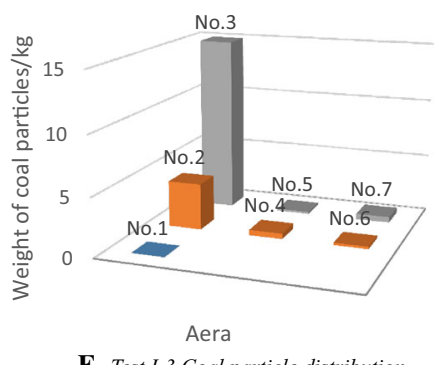

F Test I-3 Coal particle distribution

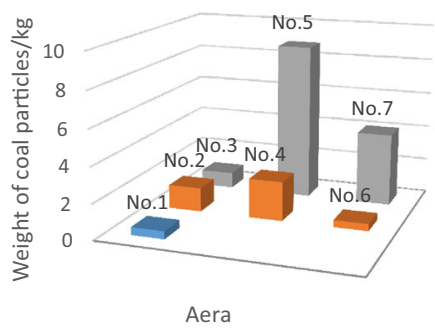

H Test II-1 Coal particle distribution

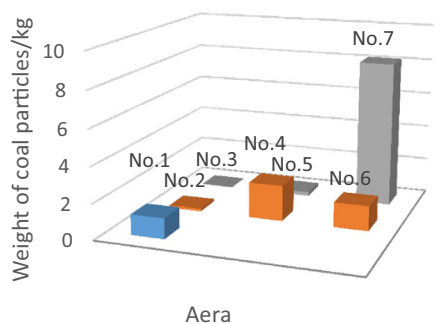

J Test II-2 Coal particle distribution

Fig. 3 Coal particle gradation curves before and after the outburst with the corresponding regional distributions 


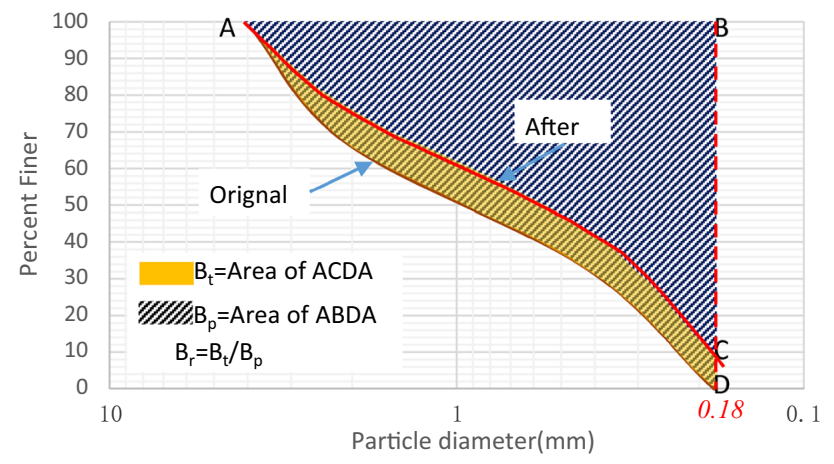

Fig. 4 Illustration of the definition of the relative breakage index

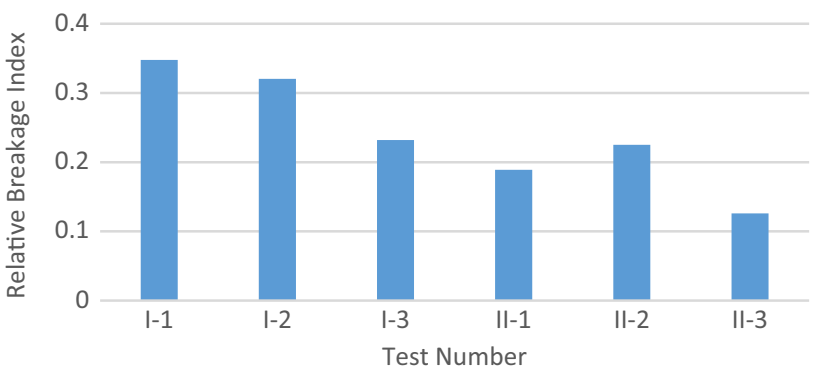

Fig. 5 The results of the relative breakage indices

larger coal piece, without artificial particle size changes. After each test, the coal particles are collected and reloaded for the next test. This process is repeated five times, and the gradation curves and relative breakage index $B_{r}$ are used to analyze the variations of the crushing effect, as shown as Figs. 6, 7, and 8.

The results of multiple tests show the following results. (1) Based on the same initial particle size, more test repetitions cause larger relative breakage indices, which illustrates the one-way accumulation of the crushing effect. Moreover, $B_{r}$ increases approximately linearly, as shown as Fig. 8. (2) The trend of the relative breakage index increment declines gradually, which indicates that further fragmentation becomes increasingly difficult. Therefore, the coal particles are subjected to crushing effects during the outburst process. Moreover, the higher the degree of fragmentation, the more difficult it is to cause further crushing and the greater the crushing work required to achieve the same degree of crushing.

\section{Conclusions}

A number of coal and gas outburst tests were performed using a laboratory test device. The influence of the initial particle size and grading on the crushing effect, the evolution law of the crushing effect for multiple outburst tests,
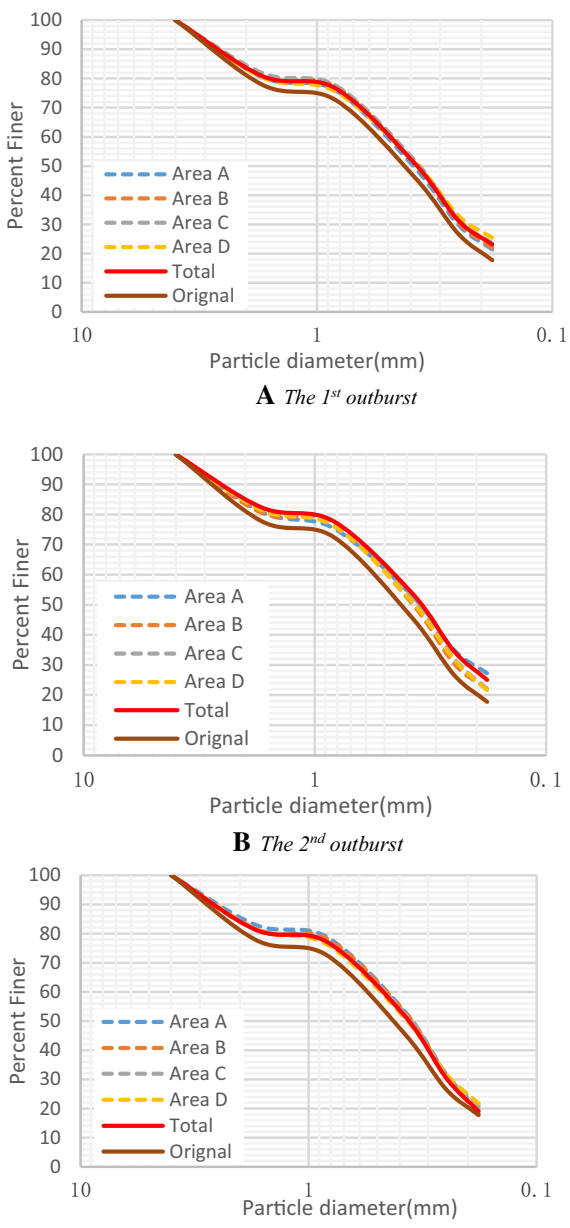

C The $3^{\text {rd }}$ outburst
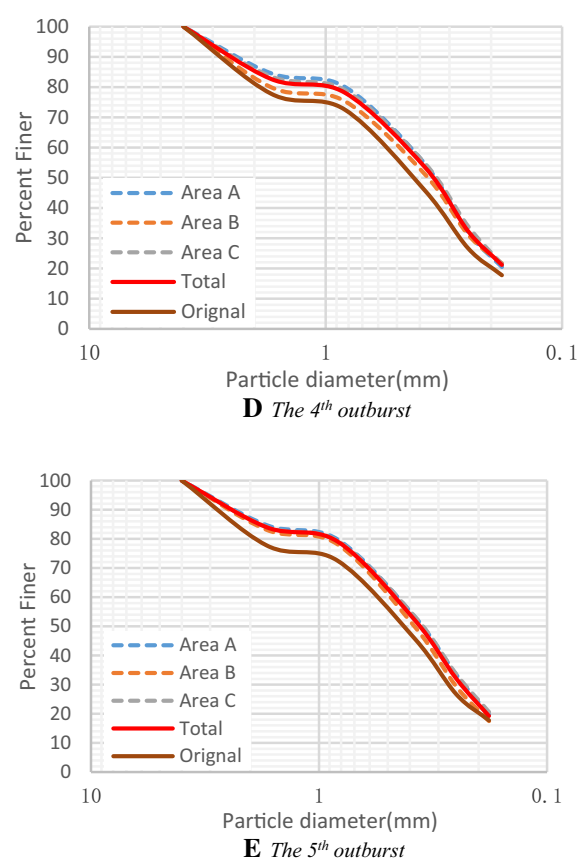

Fig. 6 Gradation curves for the cumulative crushing tests 


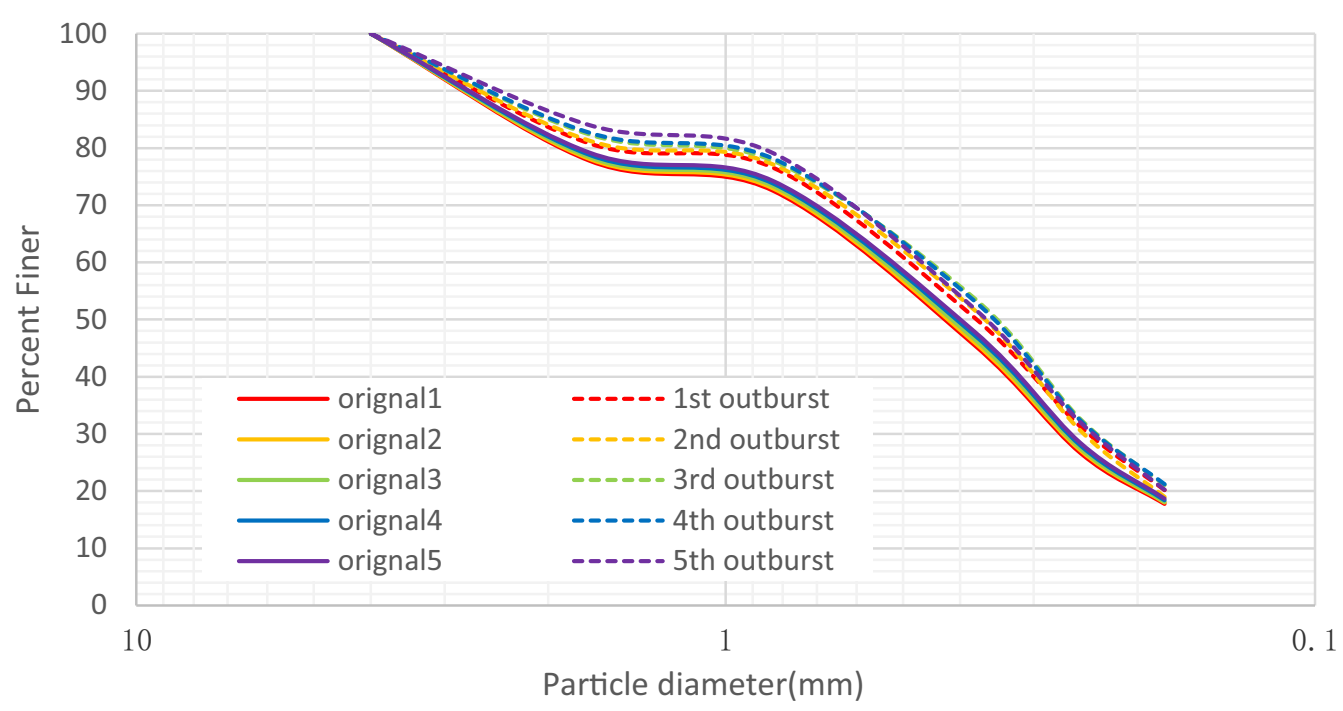

Fig. 7 Coal particle gradation curves of each outburst

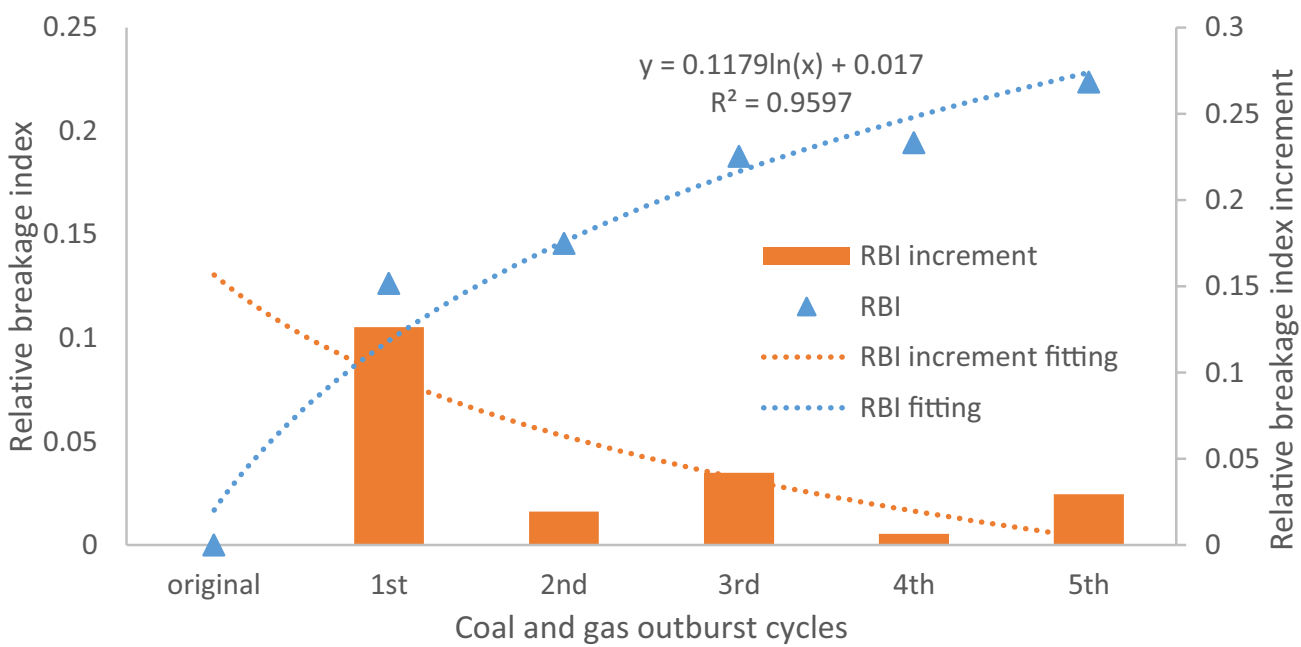

Fig. 8 Relative breakage index

and the spatial distribution of the coal that was thrown out during the outburst were all studied. The following conclusions were obtained.

(1) An energy balance equation for coal and gas outburst is established. It is believed that the energy of the coal and gas outburst is primarily from the expansion energy of the gas and the elastic potential of the coal, which is transferred to the crushing and transport work of the coal that is thrown out.

(2) The coal samples with smaller particle sizes accumulate more gas internal energy over the same limited time, which leads to more coal mass being thrown out and at greater distances. In addition, smaller particle sizes have a worse crushing effect than larger particles.
(3) The crushing effects are significantly impacted by the initial gradation of the coal particles. Coal with a good grading has a worse crushing effect. Coal samples with a uniform particle size generally have a poor gradation and are more likely to be broken.

(4) As the number of repeated tests increases, the relative breakage index $B_{r}$ increases, meaning the crushing effect continues to accumulate. However, the increment of the relative breakage index decreases after each test, showing that further fragmentation becomes increasingly difficult.

Acknowledgements This research was financially supported by the National Natural Science Foundation of China (No. 51434003), the Project of the Ministry of Emergency Management of the People's Republic of China (sichuan-0011-2018AQ), and the Department of Science and Technology of Sichuan Province (19YYJC2854). 
Author's contributions XW conceived the study, wrote the manuscript and agreed to serve as the author responsible for contact to ensure communications. JX and $\mathrm{WN}$ designed the experiments and provided suggestions. QY performed the experiments. TZ analyzed the data. YP reviewed and revised the manuscript.

\section{Compliance with ethical standards}

Conflict of interest The authors declare that they have no conflict of interests.

Open Access This article is distributed under the terms of the Creative Commons Attribution 4.0 International License (http://crea tivecommons.org/licenses/by/4.0/), which permits unrestricted use, distribution, and reproduction in any medium, provided you give appropriate credit to the original author(s) and the source, provide a link to the Creative Commons license, and indicate if changes were made.

\section{References}

Esterle J, Kolatschek Y, O'Brien G (2002) Relationship between in situ coal stratigraphy and particle size and composition after breakage in bituminous coals. Int J Coal Geol. https://doi.org/10. 1016/S0166-5162(01)00077-5

Guo LW, Yu QX, Jiang CL, Wang K (2000) Testing study on the variation of coal temperature during the process of coal and gas outburst. Chin J Rock Mech Eng 19:366-368

Gupta V, Sun X, Xu W et al (2017) A discrete element method-based approach to predict the breakage of coal. Adv Powder Technol 28:2665-2677. https://doi.org/10.1016/j.apt.2017.07.019

Hardin B (1985) Crushing of soil particles. J Geotech Eng 111:1177-1192. https://doi.org/10.1061/(ASCE)07339410(1985)111:10(1177)

Hu QT, Zou YH, Wen GC, Zhao XS (2007) New technology of outburst danger prediction by gas content. J China Coal Soc 32:276-280

Hu QT, Zhou SN, Zhou XQ (2008) Mechanical mechanism of coal and gas outburst process. J China Coal Soc 33:1368-1372

Jiang C, Yu Q (1996) Rules of energy dissipation in coal and gas outburst. J China Coal Soc 21:173-178

Krajcinovic D, Mastilovic S (1995) Some fundamental issues of damage mechanics. Mech Mater 21:217-230. https://doi.org/10. 1016/0167-6636(95)00010-0

Nie W, Yang HW, Zhang HL et al (2016) Bayesian network-based model for assessing the intensity of outbursts of coal and gas. Int J Oil Gas Coal Technol 13:200-213. https://doi.org/10.1504/ IJOGCT.2016.10000264

Oberholzer V, van der Walt J (2009) Investigation of factors influencing the attrition breakage of coal. J S Afr Inst Min Metall 109:211-216

Sahoo R (2005) Study of the commanution characteristics of coal by single particle breakage test device. Part Sci Technol 23:285-296. https://doi.org/10.1080/02726350590955921
Sahoo R (2006) Review: an investigation of single particle breakage tests for coal handling system of the gladstone port. Powder Technol 161:158-167. https://doi.org/10.1016/j.powtec.2005.09. 001

Sahoo R, Roach D (2005) Quantification of the lump coal breakage during handling operation at the gladstone port. Chem Eng Process Process Intensif 44:797-804. https://doi.org/10.1016/j. cep.2004.09.004

Shi FN (2016) A review of the applications of the JK size-dependent breakage model: part 1: ore and coal breakage characterisation. Int J Miner Process 155:118-129. https://doi.org/10.1016/j. minpro.2016.08.012

Shi FN, Liu HP, Rodrigues S et al (2018) Lithotype-based modelling and simulations of coal degradation conditioned by both high and low energy breakage. Fuel 232:405-414. https://doi.org/10. 1016/j.fuel.2018.05.125

Tavares LM, King RP (1998) Single-particle fracture under impact loading. Int J Miner Process 54:1-28. https://doi.org/10.1016/ S0301-7516(98)00005-2

Wei F, Shi G, Zhang T (2010) Study on coal and gas outburst prediction indexes base on gas expansion energy. China Coal Soc 35:95-99

Wen GC (2003) Study of coal and gas outburst energy. Min Saf Environ Prot 30:6-9

Wu X (2010) Research on simulation experiments on coal and gas outburst in various particle size. Chongqing University, Chongqing

Xie XG, Feng T, Wang Y, Huang SY (2010) The energy dynamic balance in coal and gas outburst. J China Coal Soc 35:1120-1124

Xie WN, He YQ, Wang YJ et al (2017) Effect of fine particles on the breakage behavior of coarse coal in the hardgrove Mill. Int $\mathrm{J}$ Coal Prep Util 37:326-338. https://doi.org/10.1080/19392699. 2016.1183659

Xu J, Tao Y, Yin G (2008) Development and application of coal and gas outburst simulation test device. J Rock Mech Eng 27:8

Yang DD, Chen YJ, Tang J et al (2018) Experimental research into the relationship between initial gas release and coal-gas outbursts. J Nat Gas Sci Eng 50:157-165. https://doi.org/10. 1016/j.jngse.2017.12.015

Zhang G (2008) Ventilation Safety Science. Xuzhou

Zhao YX, Jiang YD, Tian SP (2010) Investigation on the characteristics of energy dissipation in the preparation process of coal bumps. J China Coal Soc 35:1979-1983

Zheng ZM (2004) The mechanism of coal and gas outburst from the view of quantity and dimensional analysis. In: Collect Work ZHENG Zhemin, pp 382-392

Zhou SN, Lin BQ (1999) The theory of gas flow and storage in coal seams. China coal industry publishing house, Beijing

Zhou JW, Liu Y, Du CL, Liu SY (2017) Effect of the particle shape and swirling intensity on the breakage of lump coal particle in pneumatic conveying. Powder Technol 317:438-448. https://doi. org/10.1016/j.powtec.2017.05.034 